\title{
Single Particle Atomic Force Microscopy
}

\author{
Alexei Tivanski
}

University of Iowa, Iowa City, Iowa, United States

Depending on the source and relative humidity, aerosols can have different compositional, morphological, and viscoelastic properties. Significant uncertainties remain over our ability to accurately predict the overall aerosol effect on the climate and atmosphere. One reason originates from significant particle-toparticle variability of the aerosol's properties and the inherent size limitations present in current experimental techniques towards studies of submicrometer-sized particles. Studies that determine these properties at a single particle level with submicrometer spatial resolution can provide considerable information on the behavior of aerosols over a broad range of applications including atmospheric and materials science. Herein, we present several atomic force microscopy (AFM) based methodologies developed and validated on a series of model sea spray aerosol relevant systems that provide the capability to quantify the 3D morphology, growth factor, phase state, viscoelastic properties and surface tension of aerosol particles on a single particle basis as a function of relative humidity. Model compounds include various inorganic salts, organic acids, saccharides, lipopolysaccharide, and several inorganic-organic binary chemical mixtures at different mass ratios. We establish quantitative framework using AFM force spectroscopy towards differentiation of the physical phase states of individual particles and quantification of the surface tension of individual deliquesced droplets without prior knowledge of their chemical identity. Furthermore, we introduce the ability to semi-quantitatively assess the phase states of individual substrate deposited particles based on a relatively fast height imaging, irrespective of the microscopy technique used, which can then subsequently be further validated by more quantitative AFM force spectroscopy. The methods are general and can be readily applied to a wide range of nano- and microdimensional materials where the quantification of the physical phase state and viscoelastic properties under various environmental conditions (e.g. variable relative humidity, temperature) is required.

\section{References}

Ray, K. K; Lee, H. D.; Gutierrez, M.; Chang, F.; Tivanski, A. V., Correlating 3D Morphology, Phase State and Viscoelastic Properties of Individual Substrate-Deposited Particles. Analytical Chemistry. 2019, 91, 12, 7621-7630. doi: 10.1021/acs.analchem.9b00333.

Lee, H. D.; Kaluarachchi, C. P.; Hasenecz, E. S.; Zhu, J. Z.; Popa, E.; Stone, E. A.; Tivanski, A. V., Effect of Dry or Wet Substrate Deposition on the Organic Volume Fraction of Core-shell Aerosol Particles. Atmos Meas Tech, 2019, 12, 2033-2042. doi:10.5194/amt-12-2033-2019.

Lee, H. D.; Ray, K. K.; Tivanski, A. V., Solid, Semi-Solid, and Liquid Phases of Individual Submicrometer Particles Directly Probed Using Atomic Force Microscopy. Analytical Chemistry 2017, 89 (23), 12720-12726.

Lee, H. D.; Estillore, A. D.; Morris, H. S.; Ray, K. K.; Alejandro, A.; Grassian, V. H.; Tivanski, A. V., Direct Surface Tension Measurements of Individual Sub-Micrometer Particles Using Atomic Force Microscopy. The Journal of Physical Chemistry A 2017, 121 (43), 8296-8305. 